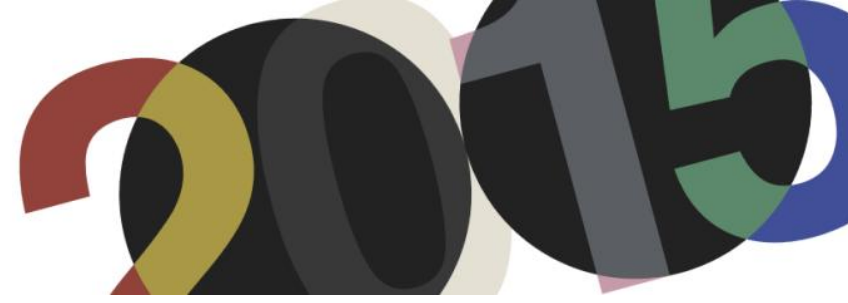

DOI: http://dx.doi.org/10.4995/LC2015.2015.1018

\title{
Le Corbusier et Christian Zervos dans Cahiers d'art
}

\author{
M.P. Sabella \\ Università degli Studi di Cagliari - DICAAR Dipartimento di Ingegneria Civile, Ambientale e Architettura
}

\begin{abstract}
The search has as purpose to notice the importance of Christian Zervos (Argostoli 1889 - Paris 1970), a greek art historian and founder of the magazine and publishing house Cahiers d'art, that lived in Paris from 1907 to the end of his life) with Le Corbusier, inserted in the contest of Cahiers d'art.

The exceptional versatility of Zervos's mind had allowed him to realize, through Cahiers d'art, a intellectual environment that exceeded the ordinary publishing house of that period, beacuse it was enchanted and nourished by all sector of knowledge. Zeros, inside the Cahiers d'art, made Le Corbusier protagonist of the section of Architecture, that submits to Sigfried Giedion. In fact since the first number of Cahiers d'art the work of Le Corbusier was broadly taken in examination. The articles related to the work of the Architect have gone since 1926 to 1954; the themes that touch these texts can be separate in four major topics: design, private house, great public buildings, painting.

La recherche a le but de relever l'importance de Christian Zervos (Argostoli 1889-Parigi 1970), historien de l'art et fondateur des Éditions Cahiers d'art, qui vécut à Paris depuis 1907 à sa mort, et Le Corbusier, insérée dans le contexte de Cahiers d'art. L'exceptionnel éclectisme de Zervos a permis de réaliser dans Cahiers d'art un excellent milieu intellectuell qu'il va au-delà des Éditions, car uni et nourri par chaque domaine de la connaissance. Dans les Cahiers d'art, Zervos rend Le Corbusier le protagoniste de la section d'architecture, soignée par Siegfried Giedion
\end{abstract}

Keywords: Cahiers d'art; Christian Zervos; projects.

\section{Introduction}

L'attention de Christian Zervos pour l'architecture et pour Le Corbusier était présent aussi pendant son activité près les Edition Albert Morancé.

En trois articles ${ }^{1}$ de Les Arts de la maison sont reportées des réflexions sur l'œuvre et sur l'influence qui exerça sur la contemporanéité, et comme celle-ci fut caractérisée par l'architecture de Le Corbusier.

En outre Zervos à l'intérieur des Cahiers d'art fit protagoniste de la section d'architecture, qu'il confie à Siegfried Giedion, Le Corbusier. Depuis le premier numéro des Cahiers l'œuvre de Le Corbusier vint amplement prise en examen.

Les articles relatifs à l'œuvre de l'architecte vont de 1926 au 1954. En totale les articles qui traitent de Le Corbusier sont vingt-et-un, deux signé par Siegfried Giedion, treize par Christian Zervos, et six par l'architecte

\footnotetext{
${ }^{1}$ Zervos C., Réalisations et projets. Le Pavillon de l'Esprit Nouveau, «Les arts de la maison», Automne \& hiver, Éditions Albert Morancé, Paris, 1925, pp. 15-18; Zervos C., "La leçon de l'Exposition des arts décoratifs et industriels de 1925", Les arts de la maison, Automne \& hiver, Éditions Albert Morancé, Paris, 1925, pp. 27-29; ZERvos C., "Réalisations et projets. Le Corbusier et Pierre Jeanneret. Intérieurs", Les arts de la maison, Printemps \& été, Éditions Albert Morancé, Paris, 1926, pp. $14-16$.
} 
même. Les sujets qui traitent ces textes peuvent se divisent en quatre macros domaines, c'est-à-dire: le design d'intérieurs, ${ }^{2}$ l'habitation privée, ${ }^{3}$ les grands bâtiments publics, ${ }^{4}$ la peinture. ${ }^{5}$

\subsection{Design d'intérieurs}

Zervos se met de façon critique à l'égard de sa contemporanéité, en ayant assisté au venir moins du «faire le nouveau». ${ }^{6}$ ${ }^{2}$ Zervos C., Architecture intérieure enquetes, «Cahiers d'art, Bulletin mensuel d'actualité artistique publié sous la direction
de Christian Zervos», n' ${ }^{\circ}$, janvier, Paris, 1926, pp. 14-15.
${ }^{3}$ Le CoRbuSIER, Notes à la suite, «Cahiers d'art, Bulletin mensuel d'actualité artistique publié sous la direction de Christian
Zervos», III, Paris, 1926, pp. 46-52; GiEDION S., "Le problème du luxe dans l'architecture moderne. A propos d'une nouvelle
construction a Garches de Le Corbusier et Pierre Jeanneret", in Cahiers d'art, Revue d'art paraissant dix fois par an-
Directeur: Christian Zervos. Peinture- sculpture-Architecture-musique-mise en scène-disques-cinéma, V-VI, Paris, 1928, pp.
254-256; Giedion Siegfried, "Le Corbusier et l'architecture contemporaine par S.Geidion", in Cahiers d'art, Revue d'art
paraissant dix fois par an- Directeur: Christian Zervos. Peinture- sculpture-Architecture-Art ancien-ethnographie-cinéma,
IV, Paris, 1930, pp. 205-215; Zervos C., "Immeuble «Clarté» a Genève par Le Corbusier et Pierre Jeanneret", in Cahiers
d'art, Revue paraissant dix fois pas an - Directeur: Christian Zervos - Peinture, Sculpture, Architecture, Art ancien,
Ethnograpgie, Cinéma, Editions «Cahiers d'art», 14, rue du Dragon, Paris (IV arrondissement), VI-VII, Paris, 1932, pp. 289-
294; Zervos C., "Jeunes architectes. A propos de leur ex position à la Galerie des "Cahiers d'art" Février-Mars 1935", in
Cahiers d'art, Revue paraissant dix fois pas an- Directeur: Christian Zervos - Peinture, Sculpture, Architecture, Art ancien,
Ethnograpgie, Cinéma, I-IV, Paris, 1935, pp.75-93.

${ }^{4}$ Zervos C., Projet pour le palais de la S.D.N. à Genève, par Le Corbusier et Pierre Jeanneret, «Cahiers d'art, Peinture, sculpture, Architecture, musique. Directeur: Christian Zervos», IV-V, Paris, 1927, pp. 175-182; Zervos C., "Qui batira le palais des nations a Genève? Étude-manifeste de «Cahiers d'art» en faveur d'un projet moderne", Cahiers d'art, Peinture, sculpture, Architecture, musique. Directeur: Christian Zervos, IX, Paris, 1927, pp. I-VIII; ZeRvos C., "Qui batira le Palais des Nations? II. La situation actuelle", Cahiers d'art, Peinture, sculpture, Architecture, musique. Directeur: Christian Zervos, IX, Paris, 1927, pp. IX-XVI; Zervos C., "Qui batira le Palais des Nations? III. Decisione del jury", Cahiers d'art, Peinture, sculpture, Architecture, musique. Directeur: Christian Zervos, n9, Paris, 1927; Zervos Christian, "Qui batira le Palais des Nations? IV. Le Conseil des Nations va statuer", Cahiers d'art, Revue d'art paraissant dix fois an-Directeur: Christian Zervos. Peinture- sculpture-Architecture-musique-mise en scène-disques-cinéma, II, Paris, 1928, pp. 84-88; LE CORBUSIER, "La Salle Pleyel. Une preuve de l'évolution architecturale", Cahiers d'art, Revue d'art paraissant dix fois anDirecteur: Christian Zervos. Peinture- sculpture-Architecture-musique-mise en scène-disques-cinéma, II, Paris, 1928, pp. 8990; Zervos C., "Un projet de centre mondial a Genéve", Cahiers d'art, Revue d'art paraissant dix fois par an-Directeur: Christian Zervos. Peinture- sculpture-Architecture-musique-mise en scène-disques-cinéma, VII, Paris, 1928, pp. 307-312; LE Corbusier, "Maison de l'Union des cootives de l'U.R.S.S. a Moscou par Le Corbusier et Pierre Jeanneret", Cahiers d'art, Revue d'art paraissant dix fois par an-Directeur: Christian Zervos. Peinture- sculpture-Architecture-musique-mise en scène, IV, Paris, 1929, pp.162-163; Zervos C., "Pour la création a Paris d'un Musée des artistes vivants", Cahiers d'art, Revue d'art paraissant dix fois par an-Directeur: Christian Zervos. Peinture- sculpture-Architecture-Art ancien-ethnographiecinéma, I, Paris, 1930, pp. 337-339; Zervos C., "Pour la création a paris d'un musée des artistes vivants(II). Réponse et projet d'aménagement et d'organisation, par Le Corbusier et Pierre Leanneret", Cahiers d'art, Revue d'art paraissant dix fois par an-Directeur: Christian Zervos. Peinture- sculpture-Architecture-Art ancien-ethnographie-cinéma, $\mathrm{n}^{\circ}{ }^{1}$, Paris, 1931, pp. 1-9; Le CORBUSIER, "Projet pour la construction du palais des Soviets par Le Corbusier et Pierre Jeanneret", in Cahiers d'art, Revue paraissant dix fois pas an - Directeur: Christian Zervos - Peinture, Sculpture, Architecture, Art ancien, Ethnograpgie, Cinéma, Editions «Cahiers d'art», 14, rue du Dragon, Paris (IV arrondissement), I-II, Paris, 1932, pp. 74-77; ZeRvos C., "Le futur palais des Soviets à Moscou", in Cahiers d'art, Revue paraissant dix fois pas an - Directeur: Christian ZervosPeinture, Sculpture, Architecture, Art ancien, Ethnograpgie, Cinéma, I-IV, Paris, 1934, p.114.

${ }^{5}$ Le Corbusier, Zervos C., Les peintures révélées par Igor Grabar, «Cahiers d'art, Revue d'art paraissant dix fois par anDirecteur: Christian Zervos. Peinture- sculpture-Architecture-musique-mise en scène-disques-cinéma», X, Paris, 1928, pp.427-428; Le Corbusier, Léger, «Cahiers d'art», III-IV, Éditions Cahiers d'art, Paris, 1933, pp. 110-111; Zervos C., Expositions. Le Corbusier, peintre (Musee National d'Art Moderne), «Cahiers d'art», $29^{\circ}$ Anno, éditions «Cahiers d'art», 14, rue du Dragon, Paris, 1954, pp.5-12. 
À l'Exposition de 1925 on réprimande «d'abord la conception générale qui a réglementé son organisation. Conçue comme une manifestation éphémère, cette exposition a empêché l'artiste de travailler sur des réalités. L'effort dans le vide ne l'a pas obligé à se mettre en face de responsabilités propres à imposer à son esprit cette mesure et cette circonspection qui nous viennent des problèmes pratiques». ${ }^{7}$

Au lieu d'organiser une inutile «foire en carton-pâte, les organisateurs de l'exposition auraient pu élever dans un coin de la région parisienne une cité modèle où tous les problèmes de l'urbanisme, de l'architecture, de l'hygiène et du confort eussent été mis à l'étude sur la base des données scientifiques les plus récentes». ${ }^{8}$

Zervos rappelle l'attention sur le donnée le plus considérable de l'exposition: «l'esprit [...] qui prévaut, c'est la tendance somptuaire. Tout est destiné aux classes favorisées par la fortune. Partout un luxe provoquant, souvent du plus mauvais goût. Des objets de peu d'utilité et d'un prix exorbitant», ${ }^{9}$ et il n'est présent «aucune mesure dans les proportions. Point de goût dans le choix de la forme. Des façades vides de toute sensibilité. Nulle humanité dans l'aspect de l'ensemble». ${ }^{10}$

Pour Zervos il est répréhensible «méconnaître l'admirable leçon de l'américain Wright, toute de bon goût, de sobriété et de sens constructif» comme aussi l'avoir perdu de vue l'exemple de la «jeune architecture hollandaise, celle qui réagit contre les tendances de l'Ecole d'Amsterdam et qui, avec l'aide des pouvoirs publics, a su se manifester d'une façon étonnante» $;^{11}$ et il est encore honteux «méconnaître l'œuvre des architectes autrichiens et l'enseignement $\mathrm{du}$ grand théoricien Adolphe Loos. C'est vouloir ignorer les constructions des architectes tchécoslovaques, qui depuis vingt ans réalisent des édifices aux formes pures, débarrassées de l'ornament, et selon des règles d'une logique impeccable. C'est enfin négliger l'effort de certains architectes allemands et des constructivistes russes dont les ouvres mériteraient une étude très attentive». ${ }^{12}$

Zervos trouve beaucoup pauvre l'architecture de l'Esposition et, à l'exception du Théâtre de Perret «l'œuvre la plus consolante de l'Exposition», ${ }^{13}$ on peut citer «malgré certaines restrictions, comme œuvres modernes que le Pavillon des Renseignements de Rob. Mallet-Stevens et le villa de Le Corbusier et Pierre Jeanneret». ${ }^{14}$ En ligne générale il y n'avait pas une «Modernité vraie» ${ }^{15}$ en entendant chose différente de la «Nouveauté morphologique», ${ }^{16}$ car la plus grande partie des artistes a donné priorité à la forme.

Dans la production des meubles on peut retrouver la même tendance qu'on est relevé en architecture: «on a changé la 'forme' d'un meuble pour une autre forme souvent bien moins heureuse et bien moins pratique. Aux styles anciens on n'a opposé que des semblants de style nouveau», ${ }^{17}$ exception fait, s'entende, pour Le Corbusier, Francis Jourdain, Pierre Chareau, Marcel Guilleminault.

\footnotetext{
${ }^{6}$ Zervos C., La leçon de l'Exposition des arts décoratifs et industriels de 1925, «Les arts de la maison», Automne \& hiver, Éditions Albert Morancé, Paris, 1925, p. 27-28.

${ }^{7}$ Ivi, pp. 27.

${ }^{8}$ Ibidem.

${ }^{9}$ Ibidem.

${ }^{10}$ Ibidem

${ }^{11}$ Ibidem.

${ }^{12}$ Ibidem.

${ }^{13}$ Ibidem.

${ }^{14}$ Ibidem.

${ }^{15}$ Ibidem.

${ }^{16}$ Ibidem.

${ }^{17}$ Ivi, p. 29.
} 
Ces rares artistes ont été capables de suivre «la leçon d'économie donnée par les constructeurs d'automobiles, de wagons et de paquebots et qui ont adapté cette leçon à l'étude des portes, des fenêtres, notamment à l'étude de l'ensemble intérieur en vue de suppléer par une organisation raisonnée, aux dimensions exiguës de la demeure contemporaine». ${ }^{18}$

Mais malgré celui-ci, selon Zervos, l'avènement de l'Exposition Internationale des Arts Décoratifs et Industriels Modernes de 1925, il ne résoulut pas le problème de l'architecture et du management des intérieurs, en partageant l'opinion de quelques artistes qui «sont unanimes à proclamer la nécessité absolue d'accorder l'œuvre avec la spiritualité de la vie moderne, ses nécessités sociales, ses inventions mécaniques». ${ }^{19}$ Ils diffèrent, pour leur avis, aussi en «ce qui concerne le sacrifice de la personnalité. Quelques-uns soutiennent l'effort des sensibilités individuelles. D'autres voient dans ces sensibilités une orientation vers l'analyse et l'expression aiguüe due moi qui retarde ou paralyse l'évolution nécessaire. Ils préfèrent les créations d'ensemble qui leur paraissent mieux répondre aux besoin du présent». ${ }^{20}$

Par contre, autres préfèrent «se soumettre à une discipline collective qui impose des contructions élémentaires propres à satisfaire aux nécessités actuelles». ${ }^{21}$ Et celà, selon Zervos, «c'est pourquoi les premiers sont partisans du meuble, tandis que les seconds, inspirés de la tradition grecque, romaine et médiévale veulent remplacer le meuble par l'utilisation rationnelle des murs». ${ }^{22}$

Les nouvelles et importantes solutions autant pour l'architecture générale que pour la gestion intérieure de l'habitation, Zervos écrit, furent introduites pendant l'Exposition par Le Corbusier que «est le prophète de la standardisation à outrance. Il poursuit la création d'une maison-type ${ }^{23}$ dans lequelle «chacune des pièces ayant une fonction déterminée, elle doit comporter un outillage approprié $[\ldots]$ sur un autre plan esthétique». ${ }^{24}$

Il est pour celà qui lui définit Le Corbusier et Pierre Jeanneret «deux artistes qui comptent parmi les meilleurs novateurs d'aujourd'hui ${ }^{25}$ et il reconnaît dans le projet pour le Pavillon de l'Esprit Nouveau un instrument valide pour apporter nouvelles et importantes solutions soit du point de vue architectural soit du management de la espace intérieure.

Ainsi dans l'entretien qui Le Corbusier accordé à Zervos, intitulé Un seule corps de métier, on été examinées des sujets relatifs à la standardisation industrielle, et de la différence qu'il doit exister entre les parties de la maison et leur fonction, et surtout l'emploi du nouveau matériel de construction, le béton armé.

Le Corbusier suppose «un seul corps de métier ${ }^{26}$ en chantier, le maçon, en imaginant que «le menuisier ne met plus les pieds dans le batiment et il ne retarde plus désespérément les travaux. Ses produits n'entrant dans la maison que lors de l'emménagement, dans des murs secs» ${ }^{27}$ Pas plus donc les vieilles étagères, qui constituent les armoires, approximativement projetées mais, par cette collaboration à la distance des techniciens spécialisés,

\footnotetext{
${ }^{18}$ Ibidem.

${ }^{19}$ Zervos C., Architecture intérieure enquetes, «Cahiers d'art, Bulletin mensuel d'actualité artistique publié sous la direction de Christian Zervos», n ${ }^{\circ}$, janvier, Paris, 1926, p. 14.

${ }^{20}$ Ibidem.

${ }^{21}$ Ibidem.

${ }^{22}$ Ibidem.

${ }^{23}$ Ibidem.

${ }^{24}$ Ibidem.

${ }^{25}$ Zervos C., Réalisations et projets. Le Pavillon de l'Esprit Nouveau, «Les arts de la maison», Automne \& hiver, Éditions Albert Morancé, Paris, 1925, p. 15.

${ }^{26}$ Ibidem.

${ }^{27}$ Ivi, pp. 15-18.
} 
on pourra avoir «une porte, une fenêtre ou un casier standard» ${ }^{28}$ réalisés pour être inséré dans l'œuvre créée parfaitement par le maçon. Le rôle du projeteur sera 'seulement' de «ordonner et proportionner». ${ }^{29}$

Pour Le Corbusier le plan de la maison moderne doit être économe de la place; à chaque outil devra être assigné sa placé et donc les meubles devront être conçus pour des usages bien précis. Alors tout ceci fut possible grâce à l'emploi du béton armé, à ses qualités spécifiques de être modelé et aux les formes multiples que peut assumer.

Zervos rappelle comme le Pavillon de l'Esprit Nouveau constitue le modèle architetturale d'un style d'habitation, d'une cellule susceptible de vitalité et qui peut se développer librement dans la réalité urbaine.

Il est important de remarquer comme Zervos considère valides seulement peu études parmi ceux exposés à la Exposition internationale des arts décoratifs et industriels modernes de 1925, quand fut montré le Pavillon de l'Esprit Nouveau.
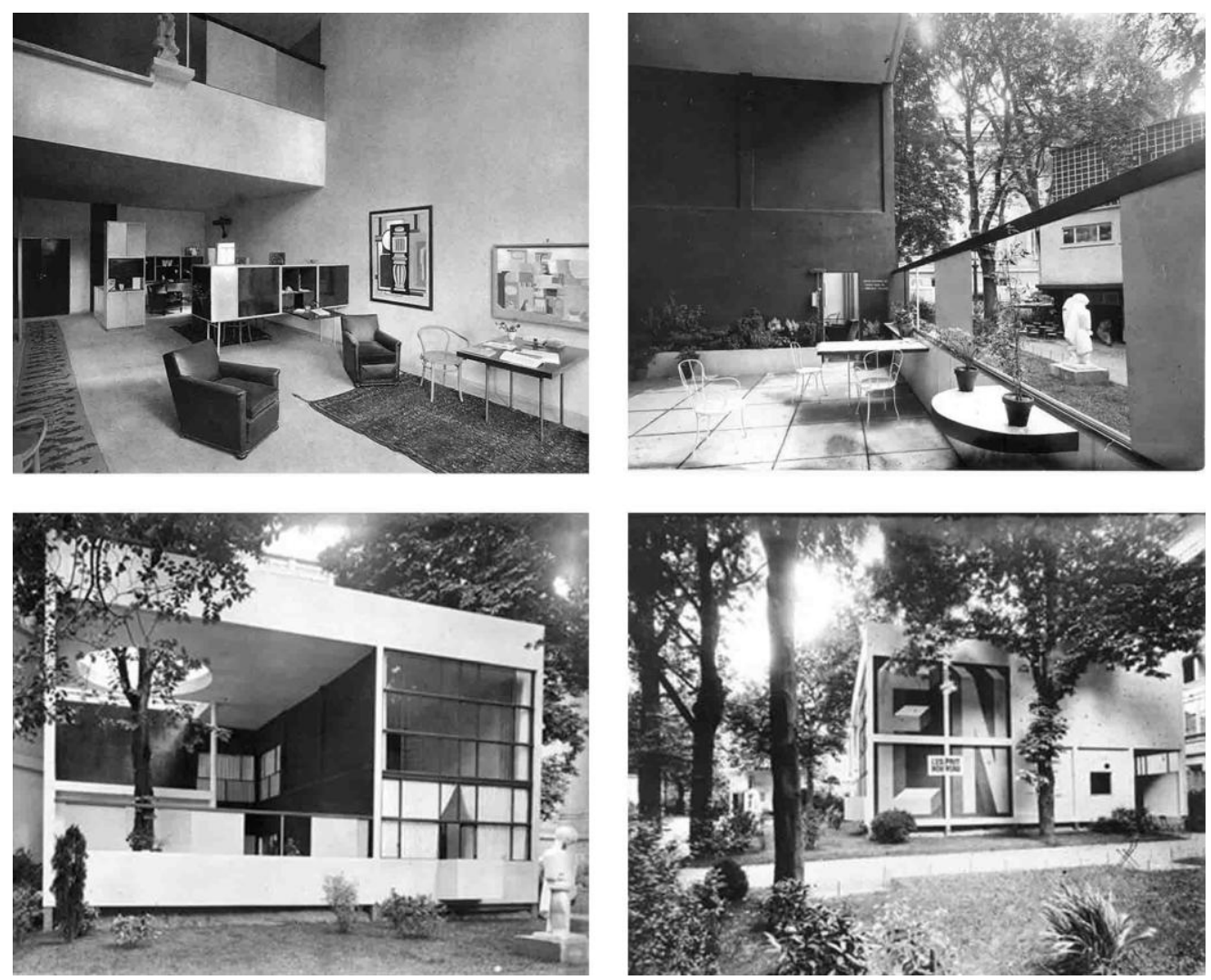

1. Pavillon de l'Esprit Nouveau , 1925.

Zervos est conscient de combien la recherche de Le Corbusier consacrât beaucoup d'attention à l'habitation privée. Un entretien relâché au éditeur grec révèle combien l'architecte fût intéressé à résoudre les problèmes relatifs à la vie dans la maison moderne. En dix-huit points Le Corbusier dédie beaucoup d'attention à la définition confort, éclairage et meubles, et en rappelant comme le verbe construire soit synonyme de faire durer, que structurer soit de résoudre les données pratiques d'un problème de comfort, et que le but de l'architecture soit prétendre émouvoir.

\footnotetext{
${ }^{28}$ Ibidem.

${ }^{29}$ Ibidem.
} 
Dans le premier point Le Corbusier déclare que «l'architecture est fonction de la lumière; c'est un phénomène plastique dans la lumière. Sans lumière, on ne voit pas. Ne voyant pas, on ne sent pas. Pas d'émotion architecturale sans lumière ${ }^{30}$ et il est dans cette circonstance qu'on situe «le phénomène architectural plus profondément encore dans le fait de la lumière: nous nous éveillons à la lumière, nous voyons, discernons, nous agissons, travaillons, pensons». ${ }^{31}$ Et à l'homme plaît que «le soleil entre dans la maison, car nous vivons sédentairement dans la grande ville ${ }^{32}$ puisque «l'homme est un animal de lumière». ${ }^{33}$

Un animal de lumière et sa «maison est un abri» ${ }^{34}$ et «plus nous devenons sédentaires, plus nous cherchons le confort»; ${ }^{35}$ par les fenêtres «le béton armé apporte des trésors de soleil» ${ }^{36}$ et «avec le béton armé, il n’y a plus de trumeau imposé. Le fenêtre peut toucher aux deux murs latéraux et les éclairer d'un bord à l'autre». ${ }^{37}$ Ainsi «nous avons conquis par la technique la base primordiale de la sensation architecturale: la lumière». ${ }^{38}$

En regardant «les murs éclairés et colorés de nos maisons ${ }^{39}$ il est possible de remarquer l'«eloquence des proportions: géométrie, épurement, classification, hiérarchie données par la couleur ${ }^{40}{ }^{4}$ q'en les réfractant sur les murs nous invite «à participer d'un phénomène architectural». ${ }^{41}$

Le Corbusier a plusieurs fois proposé la nécessité de standardiser les meubles de la maison, en proposant des étagères standardisées comme aussi tables standardisées, dont les proportions pourront avoir valeur universelle. Et non seulement, mais en les rapportant aux autres éléments d'enrichissement d'une maison, il fait une réflexion sur les tableaux, en désapprouvant la nouvelle réalité que «s'imposait dans la maison moderne: la bibliothèque à tableaux». ${ }^{42}$ Pour Le Corbusier le tableau est un panneau mobile dans lequel il faut regarder pour déchiffrer le discours qui y est écrit dedans: «un tableau est un livre, il contient quelque chose; on ne peut pas lire quarante livres à la même minute». ${ }^{43}$

Le Corbusier releve que si «on accroche de multiples tableaux, d'innombrables tableaux partout ${ }^{44}$ il se verra «finie l'eurythmie de la maison ${ }^{45}$ et l'œuvre perdra sa fonction réelle.

Élément de grande importance pour lui qui se considere «un supréme épicurien. Parce qu'un véritable épicurien recherche la jouissance pure». ${ }^{46} \mathrm{~L}$ 'architecte rappelle que

\footnotetext{
${ }^{30}$ Zervos C., Notes à la suite, «Cahiers d'art, Bulletin mensuel d'actualité artistique publié sous la direction de Christian Zervos», n³, Paris, 1926, pp. 46-52.

${ }^{31}$ Ibidem.

${ }^{32}$ Ibidem.

${ }^{33}$ Ibidem.

${ }^{34}$ Ibidem.

${ }^{35}$ Ibidem.

${ }^{36}$ Ibidem.

${ }^{37}$ Ibidem.

${ }^{38}$ Ivi, p. 47.

${ }^{39}$ Ivi, p. 49.

${ }^{40}$ Ibidem.

${ }^{41}$ Ibidem.

${ }^{42}$ Ivi, p. 52.

${ }^{43}$ Ibidem.

${ }^{44}$ Ibidem.

${ }^{45}$ Ibidem.

${ }^{46}$ Ivi, p. 51.
} 
«Epicure proposait pour but à l'homme, le bonheur, mais il le plaçait dans les jouissances de l'esprit et du cour plus encore que dans celles des sens, dans l'exercise de la raison, dans la santé du corps et de l'âme, dans les plaisirs calmes qui ne doivent ni nous priver de plaisirs plus grands ni nous causer de pénibles lendemains». ${ }^{47}$

Le Corbusier conclut ainsi: «j’ai fait mes études de construction, trés sérieusement, assez loin pour un architecte; ensuite depuis vingt années, j'ai porté un intérêt passionné à ce déroulement prestigieux d'une technique nouvelle de la construction qui nous affranchissait de servitudes séculaires». ${ }^{48}$

\subsection{Habitations privées}

En prenant en examen l'Immeuble Clarté à Genève, projet de Le Corbusier et Pierre Jeanneret, Zervos met en contraste le tentative de faire de l'immeuble «une forme d'habitation vivante». ${ }^{49}$

Une donnée significative est constituée par le fait que «l'immeuble de Genève réalise l'idée toujours mise en avant par Le Corbusier de rompre la division uniforme en étages jusque pour la vie privée». ${ }^{50}$

Le Corbusier développe typologies différentes d'habitation, qui vont «depuis le simple studio jusqu'à l'appartement de neuf pièces, et en arrive ainsi à creer des pièces de dimensions extraordinaires, occupant par exemple toute la profodeur de l'immeuble, soit quinze mètres. Ces pièces sont divisées en parties s'adaptant à divers usages et offrant des hauteurs de plafond différentes. [...] Ce sont les appartements de deux étages à deux ou trois pièces qui offrent le plus de possibilités de développement». ${ }^{51}$

La nouvelle lecture de l'espace on obtient à travers «la rupture avec la division traditionnelle en étages ${ }^{52}$ même si «l'architecte doit faire en sorte non seulement de ménager des pièces élevées, mais de les disposer sur des plans différents». ${ }^{53}$

En outre «il est étonnant de voir comment les pièces, malgré les parois vitrées, sont faciles à meubler: il existe, en effet, de grands emplacements pour les meubles qui rétablissent l'équilibre». ${ }^{54}$ Cependant le paroi vitrée reçu des critiques pas dans le demaine économique, mais «dans certaines réactions psychiques. La crainte de ne pouvoir s'isoler suffisamment de l'espace extérieur, on le sait d'expérience, existe bien plus ches l'architecte auteur du plan que chez le futur locataire. [...] La crainte des parois vitrées est étroitement liée à la notion d'architecture. My house in my castle». ${ }^{55}$

Giedion affirme que «les maisons de Le Corbusier sont la réalisation du point de vue moderne. C'est ce qui explique que la langue architecturale de Le Corbusier figure, comme élément quasi anonyme, dans l'œuvre de tant de ses contemporains». ${ }^{56}$ Selon l'historien de l'architecture «toute l'œuvre de Le Corbusier a pour but de

\footnotetext{
${ }^{47}$ Ibidem.

${ }^{48}$ Ibidem.

${ }^{49}$ Zervos C., Immeuble 'Clarté' a Genève par Le Corbusier et Pierre Jeanneret, «Cahiers d'art, Revue paraissant dix fois pas an - Directeur: Christian Zervos - Peinture, Sculpture, Architecture, Art ancien, Ethnograpgie, Cinéma», Éditions «Cahiers d'art», 14, rue du Dragon, Paris(IV arrondissement), n6-7, Paris, 1932, pp. 289-294.

${ }^{50}$ Ivi, p. 289.

${ }^{51}$ Ivi, pp. 289-290.

${ }^{52}$ Ivi, p. 291.

${ }^{53}$ Ibidem.

${ }^{54}$ Ivi, p. 294.

${ }^{55}$ Ibidem.

${ }^{56}$ GiEdion S., Le problème du luxe dans l'architecture moderne. A propos d'une nouvelle construction a Garches de Le Corbusier et Pierre Jeanneret, «Cahiers d'art, Revue d'art paraissant dix fois par an- Directeur: Christian Zervos. Peinturesculpture-Architecture-musique-mise en scène-disques-cinéma», n 5-6, Paris, 1928, pp. 254.
} 
détruire la maison en tant que 'fortesse' et d'ériger à sa place un bâtiment léger presque transparent comme ceux qu'ont rêvé les meilleurs architectes du siècle dernier lorsque, pour la première fois ils créèrent leurs merveilleuses halles en fer et en verre». ${ }^{57}$

Pour Giedion «le luxe, au sens d'autrefois, n'est plus réalisable» ${ }^{58}$ et vraiment «pour la première fois dans l'histoire nous nous trouvons devant ce singuilier phénomène: ce n'est plus la classe la plus favorisée, mais au contraire la classe la moins favorisée, qui est le facteur dominant dans la création du style moderne». ${ }^{59}$

Ce fait ne dépend pas de raisonnements sentimentaux mais se trouve dans chaque problème de la production: «nous savons très bien que l'architecture moderne n'aura acquis la stabilité et l'universalité à laquelle nous croyons, que lorsque sa plus petite cellule aura été modelée de la façon la plus méticuleuse». ${ }^{60}$

Selon Giedion l'architecture contemporaine doit être analyzée an relation avec un période spéciale, celle de la «production industrielle» ${ }^{61}$ dans lequelle est fort «la nécessité de tenir compte dans une large mesure des besoins humains», ${ }^{62}$ dont l'importance implique «que la nouvelle architecture n'est possible que par la collaboration des disciplines les plus diverses». ${ }^{63}$

À ce point Giedion reporte une définition d'Architecture de Le Corbusier, c'est-à-dire «Architecture signifie pour moi: agir par construction spirituelle». ${ }^{64} \mathrm{Il}$ s'agit d'une définition qui se complète dans le modus operandi typique de Le Corbusier qui «a su tirer parti du squelette de béton armé transmis par la science. Nous ne songeons pas aux formes de ses constructions, mais à la façon dont il fit servir l'armature aux fonctions nouvelles de l'habitation». ${ }^{65}$

Pour Giedion «c'est dans cette adaptation de la sèche construction de béton armé à l'élaboration de l'habitation moderne réclamée par l'époque ${ }^{66}$ qu'il réside l'élément fécond de l'œuvre de Le Corbusier, pour lequel est d'importance capitale que l'on renoncé «une fois pour toutes à ces murailles de mœllons entassés à la main, et qui font de non maisons d'effroyables prisons», ${ }^{67}$ en soulignant que «le facteur décisif n'est pas le progrès dans les formes, mais la méthode de construction». ${ }^{68}$

En ce qui concerne les méthodes constructives «l'armature qui s'est substituée aux murailles portantes permet un afflux de lumiere jusqu'ici inconnu ${ }^{69}$ en rendant possible un innovant accord entre «les exigences de la biologie qui s'accordent avec celles de la construction». ${ }^{70}$

\footnotetext{
${ }^{57}$ Ibidem.

${ }^{58}$ Ibidem.

${ }^{59}$ Ibidem.

${ }^{60}$ Ibidem.

${ }^{61}$ GiEdion S., Le Corbusier et l'architecture contemporaine par S. Geidion, «Cahiers d'art, Revue d'art paraissant dix fois par an. Directeur: Christian Zervos. Peinture, Sculpture, Architecture, Art ancien, Ethnographie, Cinéma», n²4, Paris, 1930, pp. 205-212.

${ }^{62}$ Ivi, p. 205.

${ }^{63}$ Ibidem.

${ }^{64}$ Ibidem.

${ }^{65}$ Ibidem.

${ }^{66}$ Ibidem.

${ }^{67}$ Ibidem.

${ }^{68}$ Ibidem.

${ }^{69}$ Ivi, p. 206.

${ }^{70}$ Ibidem.
} 
Maintenant la fenêtre prend «une importance qu'elle n'avait jamais eue. Tout naturellement, les constructions à armature de béton armé ou de fer, permettent des baies larges ou même, selon la position des piliers, des ouvertures occupant toute la largeur de la façade. [...] De la conception générale de la nouvelle construction, Le Corbusier tire l'idée de la fenêtre horizontale coulissante qui peut glisser dans les ouvertures correspondantes». ${ }^{71}$

Autre rappel à un des points de l'architecture de Le Corbusier est donné par «l'adaptation de la construction à armature aux fonctions nouvelles de l'habitation $»^{72}$ que «comporte également une transformation rationnelle du toit et l'utilisation de l'espace laissé libre par les piliers du rez de chaussée» ${ }^{73}$ et enfin «l'articulation du cube rigide et massif, telle que l'a tentée Le Corbusier dans la Villa De Monzie à Garches». ${ }^{74}$
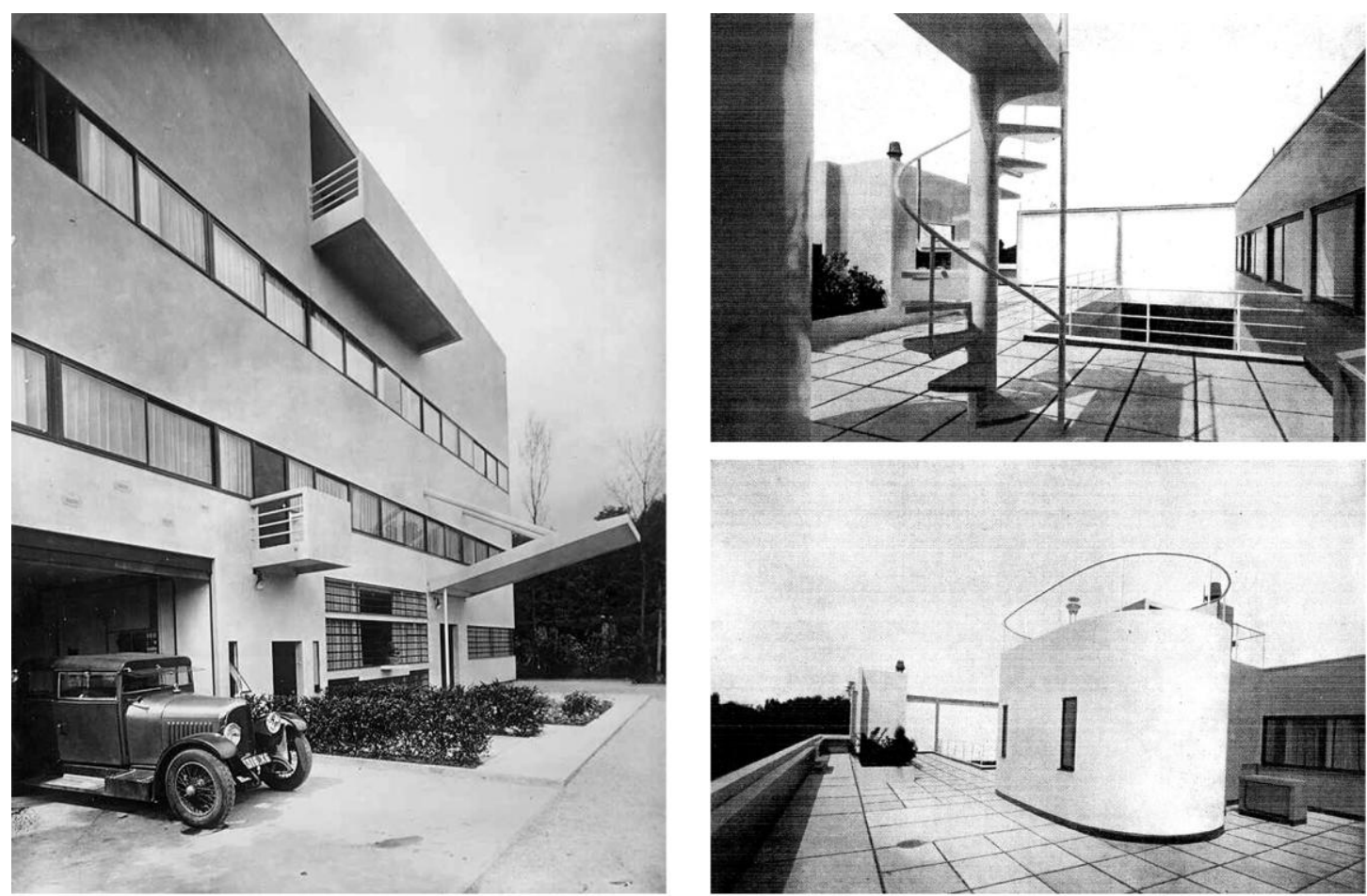

2. Villa De Monzie à Garches, 1928.

Giedion définit cette villa «un essai consistant à alléger non seulement la base, mais encora la partie supérieure et à laisser largement pénétrer l'air par la façade qui n'est plus compacte». ${ }^{75}$

Pour Giedion, Le Corbusier réalise «une architecture planante». ${ }^{76}$ Tremper son architecture demande à l'homme la création de «un nouveau sens de l'équilibre. Les recoupement, les interpénétrations, aspirent le monde extérieur». ${ }^{77}$ Et les terrasses et les ponts qui entourent presque le volume de la maison «sont du luxe. Mais en même temps leur réalisation exprime le fait que la vie privée, fermée, perd de plus en plus sa justification». ${ }^{78}$

\footnotetext{
${ }^{71}$ Ibidem.

${ }^{72}$ Ibidem.

${ }^{73}$ Ivi, pp. 206-207.

${ }^{74}$ Ibidem.

${ }^{75}$ Ivi, p. 211.

${ }^{76}$ Ibidem.

${ }^{77}$ Ibidem.

${ }^{78}$ Ibidem.
} 
Le luxe de la Villa à Garches consiste dans un «luxe de volume d'air, qui par son harmonie réalise la nouvelle conception. C'est l'exprérience hardie d'un visionnaire qui est ici réalisée; et cette expérience nous fait apercevoir le seul moyen qui permette aujourd'hui de faire du luxe une force productive». ${ }^{79}$

«Toutes les maisons de Le Corbusier ont pour but de créer un habitat pour les habitudes de vie en voie de formation ${ }^{80}$ comme aussi Villa Savoye. Le problème qui Le Corbusier se met c'est toujours d' «ouvrir la maison, cette maison qui se ferme craintivement, et créer de nouvelles possibilités de liaison entre l'intérieur et l'extérieur». ${ }^{81}$ Contrairement à les précédentes villes de Le Corbusier, construites sur un terrain resserré, Villa Savoye possède une place spéciale puisque se trouve complètement isolée. Le problème à résoudre ici «pour tout homme ayant quitté son travail et cherchant à reprendre contact avec la nature organique, avec les plantes, le ciel et le paysage». ${ }^{82}$
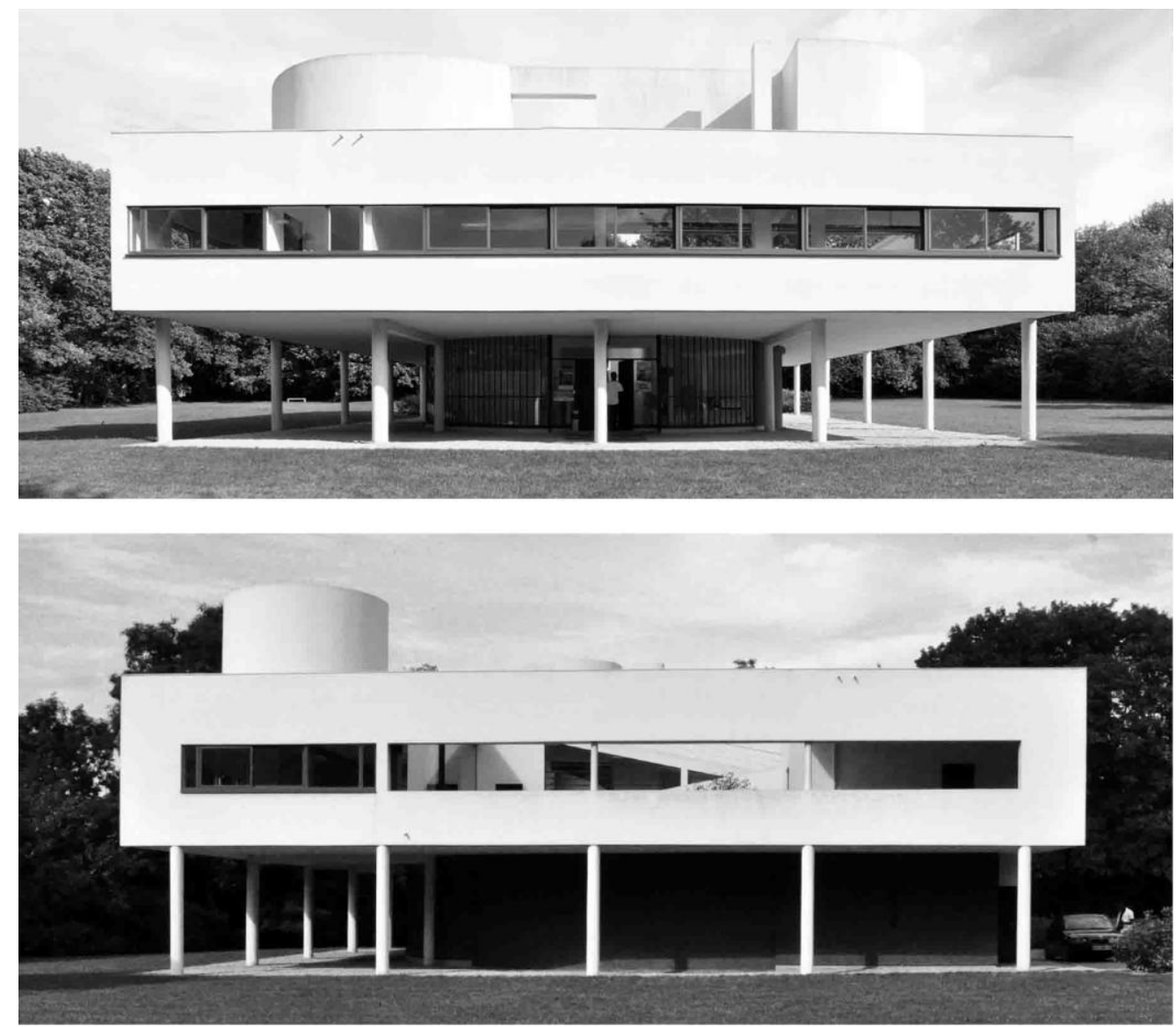

3. Villa Savoye, 1929.

Il est important de souligner comme le «notre attitude est toute différente aujourd'hui vis-à-vis de la nature. Il s'agit moins de s'adapter passivement au sol lui-meme que de s'unir avec tout le paysage: avec le ciel, et surtout avec l'air». ${ }^{83}$

\footnotetext{
${ }^{79}$ Ibidem.

${ }^{80}$ Ivi, p.212.

81 Ibidem.

82 Ibidem.

${ }^{83}$ Ibidem.
} 
«La maison repose sur pilotis et les véritables appartements se trouvent au premier étage»; ${ }^{84}$ le particulier «auquel est destinée la construction, aspire à dominer le paysage plutôt qu'à se trouver en proximité des arbres et des plantes. Il veut jouir du panorama, du vent et du soleil». ${ }^{85}$

Giedion décrit la maison comme «un cube reposant sur pilotis. Le cube n'est pas une masse pleine, mais évidé dans la direction du Sud-Est et du Sud-Ouest, et de telle sorte que le soleil levant n'effleure pas seulement les surfaces extérieures mais pénètre jusque dans l'intérieur de la maison». ${ }^{86}$

En outre «il n’y a pas de façade, pas de devant ni de derrière, car la maison s'ouvre de tous côtés». ${ }^{87}$

\subsection{Grands bâtiments publics}

Le projet pour le Palais de la Société des Nations à Genève, conçu par Corbusier et Pierre Jeanneret est «une leçon très complète d'architecture moderne. De nombreux problèmes d'ordre esthétique et d'ordre technique et pratique s'y trouvent résolus». ${ }^{88}$

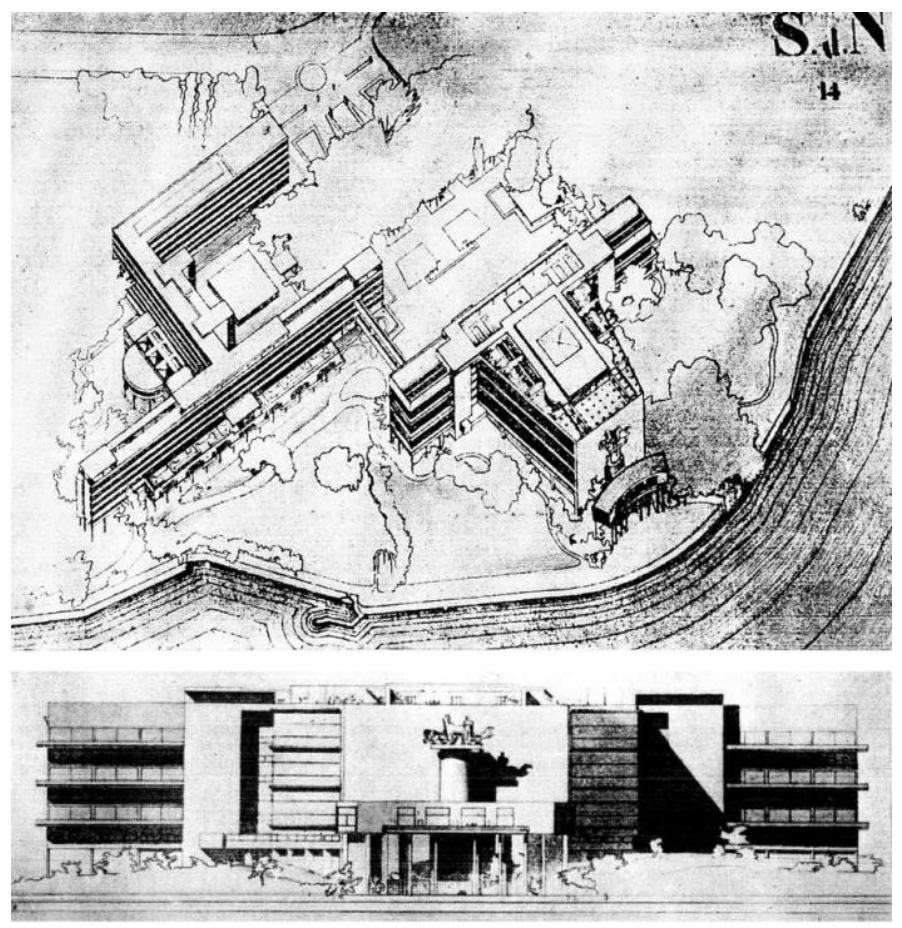

4. Projet pour le Palais de la Société des Nations à Gèneve, 1927.

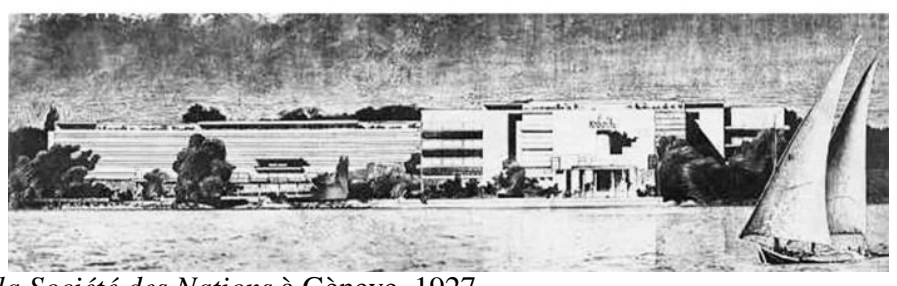

\footnotetext{
${ }^{84}$ Ibidem.

${ }^{85}$ Ibidem.

${ }^{86}$ bidem.

${ }^{87}$ Ibidem.

${ }^{88}$ Zervos C., Progetto per il Palazzo della S.D.N. a Ginevra, a cura di Le Corbusier e Pierre Jeanneret, «Cahiers d'art. Peinture, sculpture, architecture, musique. Directeur: Christian Zervos», IV-V, Paris, 1927, p. 175.
} 
Avant tout les deux architectes ont analysé soigneusement «le site où ce Palais doit s'élever, contrairement aux habitudes des architectes qui réalisent sur le papier des monuments passe-partout ${ }^{89}$ pour rendre le projet «partie intégrante du site». ${ }^{90}$

Du point de vue topographique ce projet «évite les espaces morts en mettant les bâtiments au travers de tout le terrain». ${ }^{91}$ En outre «s'oppose au 'Système fermé'; procède par 'redents' très ouverts à la vue, à la lumière, au soleil ${ }^{92}$ et «ne touche pas au terrain, évite le déplacement des terres en vue du terrassement ou en vue des fouilles». ${ }^{93}$

Les bâtiments «étant tous au même niveau et occupant des places très différentes du terrain accidenté, ils seraient construits sur des poteaux 'pilotis' de hauteurs variables. Les vastes espaces couvertes ainsi crèés sont utilisés pour faire des garages» ${ }^{94}$ et la circulation des autos «se fait à sens unique». ${ }^{95}$

Élément capital du bâtiment fut la fenêtre et cette «solution technique, fenetre à double vitrage coulissante latéralement et fermeture hermétique par excentrique serrant automatiquement les châssis contre une nervure médiane, est assurée par un type existant déjà et appliqué avec succès». ${ }^{96}$

L'éditeur grecque met en évidence comme «le bâtiment de la Grande salle s'avance en proue vers le lac, jusqu'au bord de l'eau. La vue de la terrasse est donc tout à fait exceptionnelle. La salle est étudiée à fond au point de vue acoustique, aération, ventilation, chauffage, purification de l'air». ${ }^{97}$

Le système d' «aération ponctuelle' qu'ils se sont proposés d'appliquer dans la grande salle, chauffe de l'air à $18^{\circ}$ [...] pénètre dans le locaux de purification et d'ozonification, il est, soit réchauffé, soit réfrigéré, de façon à être maintenu constamment à $18^{\circ}$, été comme hiver». ${ }^{98}$

Élément innovant se revela celui-là «d'accord avec les travaux de Gustave Lyon, de la Salle Pleyel»; ${ }^{99}$ Le Corbusier et Pierre Jeanneret ont conçu une salle «d'après les lois rigoureuses de l'acoustique, qui permettent de diriger les ondes sonores et de faire état de murs projecteurs et de murs réfléchisseurs». ${ }^{100}$

Avec la Salle Pleyel on était atteint «la Chimère de l'acoustique avait été poursuivie depuis l'antiquité par les voies incertaines et, en l'occurence, inopérantes de l'empirisme. Mais, aujourd'hui, le monde de la construction sait dorénavant qu'en peut résoudre scientifiquement l'acoustique des grandes salles». ${ }^{101}$

L'innovation, nécessaire surtout en architecture, c'est fondamental et sert à desserrer «pour le monde crédule, la vérité [...]. Mais voici qu'aujourd'hui, la salle Pleyel, c'est la vérité aussi, la vérité de fait, la vérité

\footnotetext{
${ }^{89}$ Ibidem.

${ }^{90}$ Ibidem.

${ }^{91}$ Ibidem.

${ }^{92}$ Ibidem

${ }^{93}$ Ibidem.

${ }^{94}$ Ibidem.

${ }^{95}$ Ibidem.

${ }^{96}$ Ivi, pp. $175-176$.

${ }^{97}$ Ivi, p. 176.

${ }^{98}$ Ibidem.

${ }^{99}$ Ibidem.

${ }^{100}$ Ibidem.

${ }^{101}$ Le CORBUSIER, La Salle Pleyel. Une preuve de l'évolution architecturale, «Cahiers d'art, Revue d'art paraissant dix fois an. Directeur: Christian Zervos. Peinture, Sculpture, Architecture, Musique, Mise en scène, Disques, Cinéma», n², Paris, 1928, pp. 89-91.
} 
fonctionnante, s'opposant à la vérité factice de l'Institut; et l'Institut s'incline devant cette nouvelle vérité irréfutable». ${ }^{102}$

Confiant en l'arriver et dans la sensibilité qui tôt ou tard devront émerger, l'architecte déclare que «il est à prèsumer que dans le monde entier, tout futur tenancier de salle exigera le maximum de sécurité acoustique. Et le nombre des salles Pleyel se multipliera». ${ }^{103}$

Il est ainsi que Le Corbusier argue sur «le valeur historique de la Salle Pleyel: sans ambiguité ni restriction, elle est vraie, comme est vrai l'avion qui vole et le poisson qui nage». ${ }^{104}$

Le Corbusier met en garde les lecteurs en déclarant que «ces nouvelles salles seront modernes. L'étant, voici la résultante tragique: impossible de dessiner, ni meme de concevoir, dans de telles salles, le moindre ordonnance dite classique: pilastres, colonnes, entablements, coupoles, moulures, bref, tout ce qui se dessine, et tout ce qui, en fait, remplit les manuels d'histoire de l'art. nous ne sommes plus ici, dans un système orthogonal rappelant l'un quelconque des systèmes architecturaux du passé; nous sommes dans un systeme biologique, où tout est plans gauches, surfaces gauches, insaisissables, incapables de recevoir le moindre système de décor orthogonal». ${ }^{105}$

Pour Zervos «il s'agit de la lutte de l'ancien contre le moderne. Cette lutte intéresse toutes les élites, parce que l'objet, le Palais de la Société des Nations, est une œuvre capitale et que l'issue de cette bataille comptera en plus ou en moins dans l'histoire de l'évolution contemporaine». ${ }^{106}$

Le tableau d'honneur du concours international pour la construction de le Palais de Genève fut rendu public dans le mois de mai 1927, accolé par «le laconique rapport du jury», ${ }^{107}$ et dans le mois suivant il eut lieu l'exposition des projets de «377 cabinets d'architecture d'Europe et d'Amerique». ${ }^{108}$

Selon Zervos «il y avait véritablement là, la somme des puissances architecturales» ${ }^{109}$ de l'époque, et «deux tiers des envois constituaient la contribution de l'académisme le plus fin comme aussi le plus plat». ${ }^{110}$

Le palmares représentait de manière admirable «dans une diversité incohérente, la totalité des manifestations architecturales d'aujourd'hui: École des Beaux-Arts de Paris, emphase archéologique italienne, distinction néoclassique scandinave, superficialité 'moderne' d'un côté de la production germanique, modernisme rationel et digne de la France d'avant-garde». ${ }^{11}$

Devant à une manifestation si vaste et différente de l'architecture, comme il pouvait être le jury capable de comprendre et évaluer un «tel éclectisme», ${ }^{112}$ et avec un palmares que «comportait neuf premiers prix, neuf

\footnotetext{
${ }^{102}$ Ivi, p. 89.

${ }^{103}$ Ibidem.

${ }^{104}$ Ibidem.

${ }^{105}$ Ivi, p. 90.

${ }^{106}$ Zervos C., Qui batira le palais des nations a Genève? Étude-manifeste de 'Cahiers d'art' en faveur d'un projet moderne, «Cahiers d'art, Peinture, sculpture, Architecture, musique. Directeur: Christian Zervos», n 9 , Paris, 1927, p. I.

${ }^{107}$ Ivi, p. II.

${ }^{108}$ Ibidem.

${ }^{109}$ Ibidem.

${ }^{110}$ Ibidem.

${ }^{111}$ Ibidem.

${ }^{112}$ Ibidem.
} 
deuxième prix, neuf troisième prix, contrairement au programme qui prévoyait: un première prix, deux deuxièmes prix, un troisième, quatrième, cinquième, sixième et septième prix». ${ }^{113}$

Zervos énumère les événements qui caractérisèrent le cours des soixante-quatre séances du jury, en relevant comme à la soixante-troisième le projet numéro 273, celui de Le Corbusier et Pierre Jeanneret «était mis au premier rang et recommandé avant tout autre à l'attention du Conseil. Pendant toute le durée des déliberations qui furent extraordinairement laborieuses, le projet Le Corbusier fut le seul qui réunit constamment les suffrages de quatre architectes de reputation mondiale qui siégeaient au jury». ${ }^{114}$

Ce qui pressait Zervos était l'imprévu qu'il rencontra au dernier moment et qu'il concernait une règle contenu à l'intérieur du statut qui semblait interdire l'usage des «moyens mécaniques». ${ }^{115}$ Les plans de Le Corbusier et Pierre Jeanneret, «dessinés à l'encre comme tous les autres, avaient été reproduits pour plus de netteté, à la presse d'imprimerie» ${ }^{116}$ et ceci fournit l'occasion de contestation de la part de quelque juré; malgré cela «il devenait difficile de tirer hors de pair le projet Le Corbusier, et comme aucun des autres ne s'imposait, le jury se tira d'affaire en classant les meilleures, mettant ainsi la Société des Nations dans un embarras dont elle n'est pas près de sortir». ${ }^{117}$

Pour Zervos on peut se demander s'il est admissible «qu'un projet de haute valeur ait été ainsi refoulé pour des raisons qui n'ont rien à voir avec l'architecture». ${ }^{118}$

Un autre aspect important du problème du projet 273 ère constituée par le coût: «le projet 273 (Le Corbusier et Pierre Jeanneret) indique la somme de 12 milions; les autres fournissent tous les chiffres de 13 millions», ${ }^{119}$ seuil déclaré explicitement dans l'avis du concours.

L'unique objection enlevé fut celle qui mettait en évidence comme «si huit projets coûtent de 17 à 50 millions, comment se peut-il qu'un projet chiffre à 12 millions? Ce projet doit être incomplet; il est incomplet». ${ }^{120}$

Le Corbusier il défendit le caractère exhaustif du projet, comme le devis qui fut rédigé par les «meilleures entreprises suisses», ${ }^{121}$ en soulignant que rester à l'intérieur des limites du concours est dû au fait qu'il concevait «l'architecture sur d'autres bases». ${ }^{122}$ Le projet en effet «correspond rigoureusement aux nécessités d'exploitation intérieure de la Société des Nations et cela par une économie caractéristique de l'espace dont l'effet est de faciliter l'usage des bâtiments et ed déterminer un cube restreint des bâtiments. Ensuite, contrairement aux autres, il contient toutes les indications de structure dans les moindres détails; si l'on voulait, l'exécution pourrait commencer dès demain, sur les plans qui sont dans les caisses, au secrétariat de la Société des Nations». ${ }^{123}$ Le Corbusier précise que si le prix est autre «c'est que [...] nos procédés de structure sont intièrement standardisés et que notre ossature de béton porte les services requis avec exactitude et économie, et que rien dans toute notre étude, n'est inutile. Ce sont là des bases entièrement neuves, conformes à tout ce qui régit le travail contemporain dans tous les domaines autres que celui de l'architecture et ces bases sont opposées

\footnotetext{
${ }^{113} I v i$, pp. II-III.

${ }^{114}$ Ivi, p. III.

${ }^{115}$ Ibidem.

${ }^{116}$ Ibidem.

${ }^{117}$ Ibidem.

${ }^{118}$ Ivi, pp. III-IV.

${ }^{119}$ Ibidem.

${ }^{120}$ Ivi, p. IV.

${ }^{121}$ Ibidem.

${ }^{122}$ Ibidem.

${ }^{123}$ Ivi, pp. V-VI.
} 
à celles qui ont servi à établir les projets coûteux où l'emphase seule a préoccupé les auteurs, au détriment le plus fatal des services de la Société des Nations». ${ }^{124}$

La commission des cinq membres, à laquelle enfin tous les pouvoirs étaient délégués, n'arriva pas à une conclusion à la fin de la session et pour éviter un rapport à l'Assemblée de 1928, pour obtenir des nouveaux crédits «faisait augmenter de $50 \%$ la somme de 13 millions fixée aux conditions du concours», ${ }^{125}$ prise de position que «bouleverser les juristes et les gens de bonne foi». ${ }^{126}$

Zervos écrit que «le projet Le Corbusier et Pierre Jeanneret occupe tous les esprits. Et cela à un tel point, que devant un danger immanent dont le sentiment pouvait bien être éveillé par la confusion des discussions de la dernière session de Genève, les élites de tous pays sont émues. Et les associations professionnelles ont décidé de faire entendre leur voix à Genève». ${ }^{127}$

Pour Zervos tel «bataille n'est pas du tout entre tels ou tels des neufs projets primés en premier. C'est une double bataille de principe: d'hégémonie (bataille diplomatique) et d'esprit ancien contre esprit moderne». ${ }^{128}$ Une bataille diplomatique qui risque ne pas trouver «un instant où seront débattues de questions d'architecture». ${ }^{129}$

Ce qui préoccupe Zervos c'est de savoir qui pourrait triompher à la dernière minute entre la stupeur générale «à la barbe de l'Angleterre et de l'Italie [...] le 'Palais Nénot' président de l'Academie des Beaux-Arts et de l'Institut de France». ${ }^{130}$

L'éditeur reporte comme «cette histoire du Palais est devenue, dans l'élite, le prétexte à proclamer l'avènement d'un esprit neuf». ${ }^{131}$

Mais le jury, composé de «Adatchi, Osuski, Sir Hilton Young, Politis, Urruta, a décidé que le Palais des Nations serait construit par Nénot, Prix de Rome, Membre de l'Institut, Prèsident du Salon des Artistes Français». ${ }^{132}$

La pensée de Nenot qu'il croit l'architecture de Le Corbusier une 'barbarie', c'est pour Zervos comme une offense aux propres temps, un outrage à l'inclinaison contemporaine et un désir évident de freiner chaque type de tension vers la recherche du nouveau.

Zervos ayant pris partie active au débat sur le Palais des Nations croit que la revue Cahiers d'art, considerant tout ce qui il est arrivé, pour sa raison d'être «se trouve fortement mêlée. [...] Revue qui, dans les œuvres de l'art, défend l'esprit moderne». ${ }^{133}$

Cependant «depuis l'ouverture de la Session de la Société des Nations à Genève, des membres du Conseil, les principales délégations de l'Asseblée, le Secrétariat général, ainsi que les autorités génevoises et la population de

\footnotetext{
${ }^{124} I v i$, p. V.

${ }^{125}$ Ibidem.

${ }^{126}$ Ibidem.

${ }^{127}$ Ivi, p. VIII.
}

${ }^{128}$ Zervos C., Qui batira le Palais des Nations? II. La situation actuelle, «Cahiers d'art, Peinture, Sculpture, Architecture, Musique. Directeur: Christian Zervos», n 9 , Paris, 1927, p. IX.

${ }^{129}$ Ibidem.

${ }^{130}$ Ibidem.

${ }^{131}$ Ivi, p. X.

${ }^{132}$ Zervos C., Qui batira le Palais des Nations? III. La situation actuelle, «Cahiers d'art, Peinture, sculpture, Architecture, musique. Directeur: Christian Zervos», nº10, Paris, 1927, p. XI.

${ }^{133}$ Zervos C., Qui batira le Palais des Nations? IV. Le Conseil des Nations va statuer, «Cahiers d'art, Revue d'art paraissant dix fois an. Directeur: Christian Zervos. Peinture, Sculpture, Architecture, Musique, Mise en scène, Disques, Cinéma», n², Paris, 1928, p. 84. 
Genéve, on été saisis par l'Union des Associations Internationales, représentée par Paul Otlet, sénateur de Bruxelles, du projet d'édifier à Genéve un centre mondial». ${ }^{134}$

Le projet du Mundaneum dessiné par Le Corbusier et Pierre Jeanneret, «Architectes à Paris, lauréats du concours du Palais des Nations», ${ }^{135}$ a été montré dans l’Université de Genève et «exposé aux délégués et au public génevois, le projet de la Cité Mondiale, qui serait à la fois Quartier Général pour les Associations, Congrès, libres mouvements internationaux et centre scientifique, documentaires, éducatif, réalisant au degré mondial et avec la coopération des organismes officiels: les cinq grandes institutions traditionnelles du Travail Intellectuel: Bibliothèque, Musée, Association scientifique, Université, Institut». ${ }^{136}$

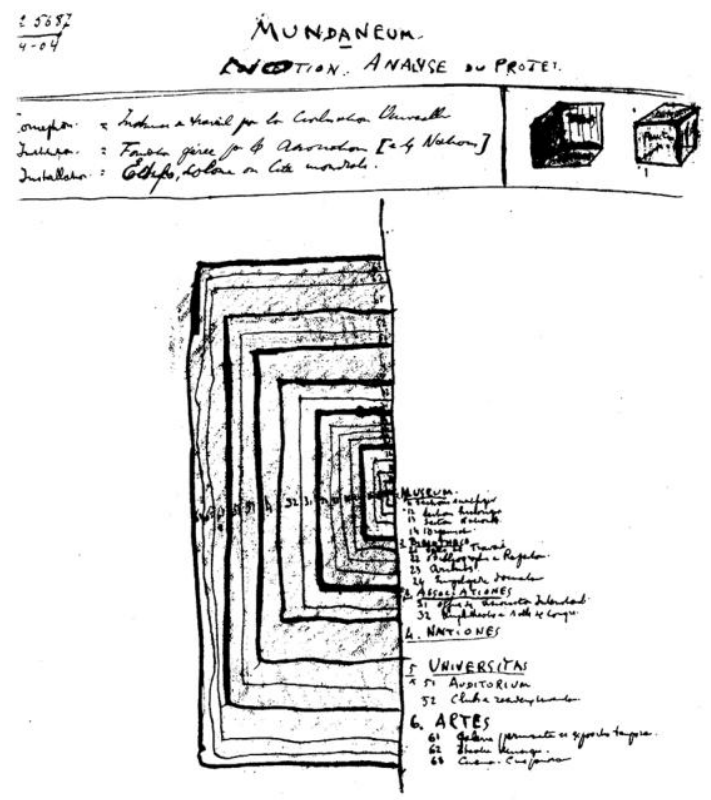

\section{Dessin du Mundaneum.}

Le Corbusier soutient que «le but du Mundaneum est d'exposer et de faire connaitre par l'écrit, par l'objet et par la parole: Comment les Hommes, de leurs humbles origines, se sont élevés jusqu'à la splendeur de leurs Génies, de leurs Héros et de leurs Saints. Comment la Terre a été dècouverte et, ses Forces ayant été soumises, presque entièrement habitée. Comment se sont élevées et les Villes, et les Nations, et le Civilisations. Comment les êtres humains, au nombre de centaines de millions, sont parvenus ò vivre en communauté sur la planète. Comment, depuis que le temps et l'espace ont été graduellement vaincus, toutes les idées et tous les actes, s'enchainent, ont leurs répercussions du Nord au Sud, de l'Orient à l'Occident, et constituent désormais une Pensée collective faite de toutes les pensées particuliéres, une Activité générale faite de toutes les pensées particuliéres, une Activité générale faite de toutes les activités spéciales. Comment, après que la Famine et la Peste durent reculer, hier, devant le Travail et la Science, à son tout la Guerre, aujourd'hui, doit céder devant une Paux voulue et organisée.

${ }^{134}$ Zervos C., Un projet de centre mondial a Genéve, «Cahiers d'art, Revue d'art paraissant dix fois par an. Directeur: Christian Zervos. Peinture, Sculpture, Architecture, Musique, Mise en scène, Disques, Cinéma», nº 7, Paris, 1928, p. 307-311. ${ }^{135}$ Ibidem.

${ }^{136}$ Ibidem. 
Comment, enfine, l'Esprit l'emportant sur la Matière, il faut que l'Idéal préside aux destinées, et que, sur la Terre aussi, il se réalise sous les formes élevées qu'ont définies les siècles: Vérité, Beauté, Bonté; Foi, Espérance et Charité; Justice et Perfection; Liberté, Égalité, Fraternité». ${ }^{137}$

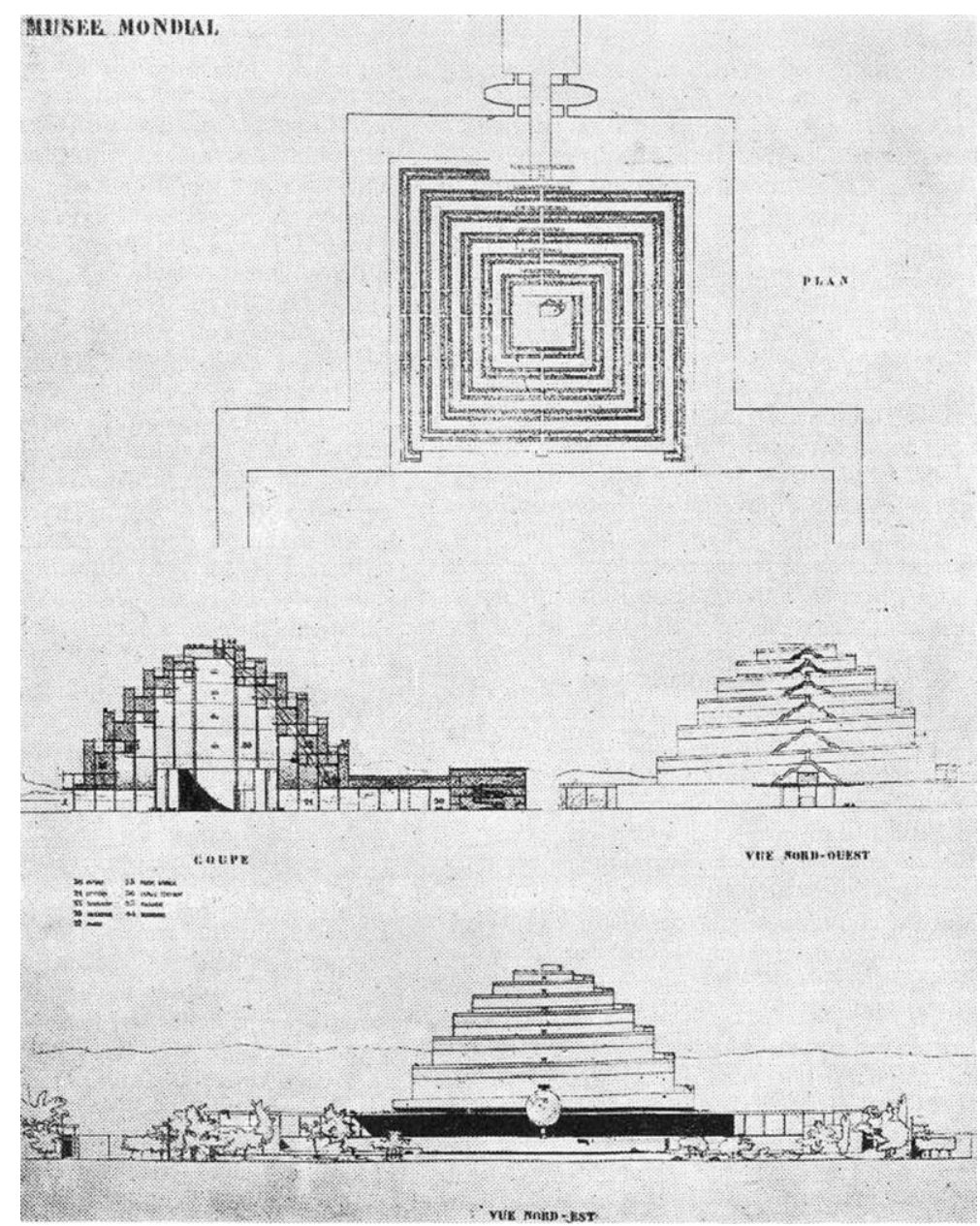

6. Projet du Mundaneum.

Le désir de Le Corbusier était que «en un point du Globe, l'image et le signification totales du Monde puissent être perçues et comprises; que ce point devienne un lieur sacré, inspirateur et coordinateur de grande idées, de nobles activités. Qu'il y soit formé un Trésos, fait de la somme des oeuvres intellectuelles, apporté comme une contribution à la Science et à l'Organisation Universelle, comme un élément de l'immense Épopée et de l'Aventure magnifique poursuivies à travers les âges par l'Humanité». ${ }^{138}$

Zervos est convaincu que «le monde trouverait là le lieu où s'élève au plus haut, le sens de la solidarité humaine, où se démontrent les conséquences des fautes et des bienfaits, où l'analyse fournit ses informations précises, où la synthèse montre les directions, où les moyens de coordination et d'harmonisation sont présents». ${ }^{139}$

\footnotetext{
${ }^{137}$ Ibidem.

${ }^{138}$ Ivi, p. 308.

${ }^{139}$ Ivi, p. 311.
} 
Dans les mêmes ans du projet pour le Palais des Nations, Le Corbusier et Pierre Jeanneret furent engagés dans l'étude pour un autre concours international, pour la construction de le Palais de l'Union des Coopératives de l'U.R.S.S. à Moscou. Les deux architectes «viennent d'être chargés de l'exécution de ce travail sur l'insistance de l'association des architectes modernes de Moscou auprès des pouvoirs». ${ }^{140}$
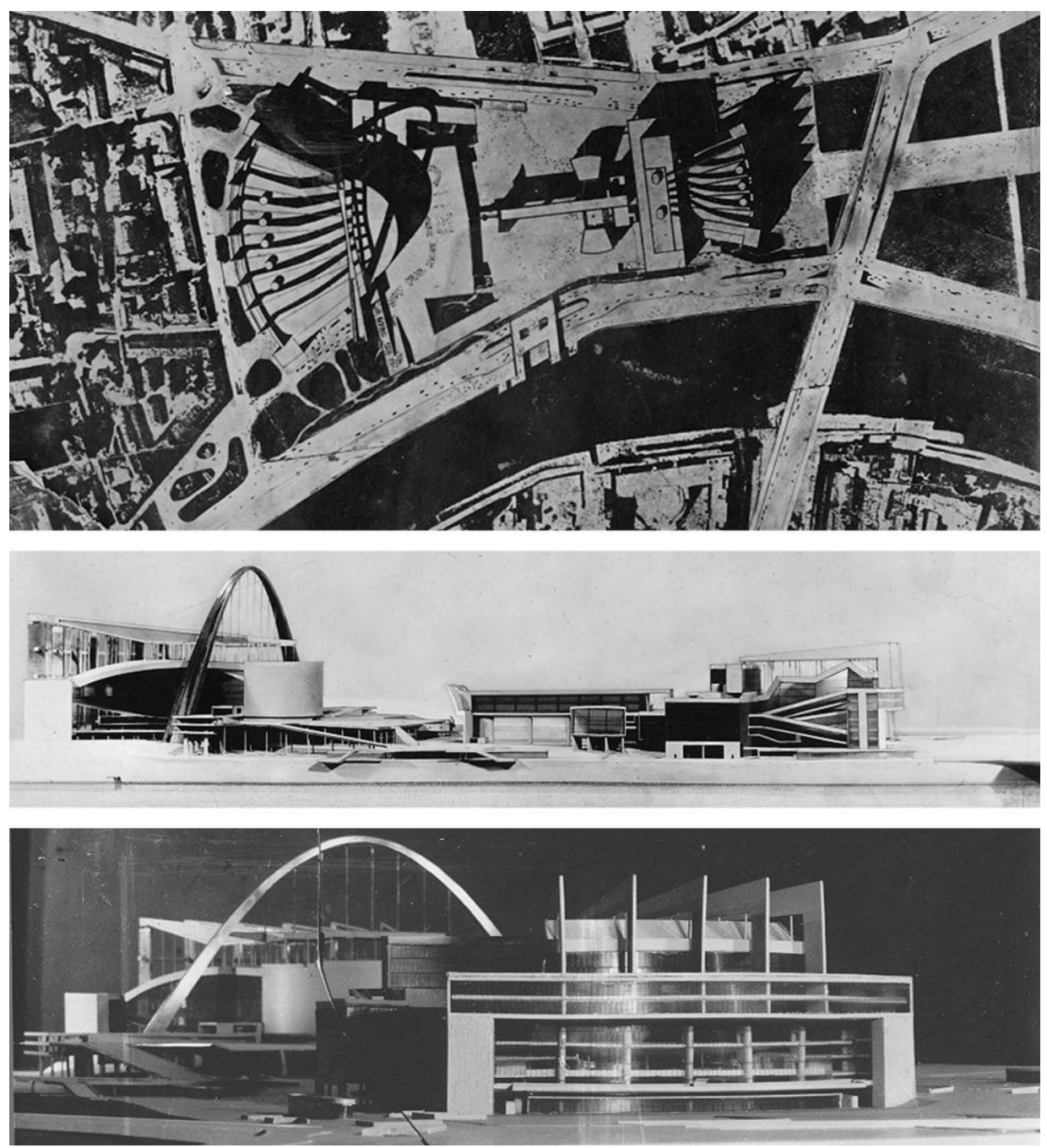

8. Projet pour la Maison de l'Union des Cootives de l'U.R.S.S.

La second phase du concours, en 1928, devait «fourni à Moscou une nouvelle série de plans émanant de cabinets d'architecture notoires d'Autriche, d'Allemagne, de France et d'Angleterre». ${ }^{41}$ Le projet de Le Corbusier et Pierre Jeanneret vint tout de suite tenu en grande considération, autant que «Le Corbusier partit pour Moscou en octobre 1928, et sur place, dut faire un second projet tenant compte très exactement des conditions locales. Enfin en février 1929 le plan définitif était adopté». ${ }^{142}$

\footnotetext{
${ }^{140}$ Zervos C., Maison de l'Union des Cootives de l'U.R.S.S. a Moscou par Le Corbusier et Pierre Jeanneret, «Cahiers d'art, Revue d'art paraissant dix fois par an. Directeur: Christian Zervos. Peinture, Sculpture, Architecture, Musique, Mise en scène», nº 4, Paris, 1929, p. 162-163.

${ }^{141}$ Ibidem.

${ }^{142}$ Ibidem.
} 
La remise donnée aux architectes des dirigeants du concours de Moscou était celle d'apporter au projet pour le Palais de l'Union des Coopératives de l'U.R.S.S. «tout ce que la technique moderne a créé d'efficace dans l'industrie du bâtiment». ${ }^{143}$

Le but était de mettre d'une manière beaucoup à l'avant-garde et parfaitement en syntonie aux demandes imposées. Le projet qui conçurent «gràce aux libertés apportées à l'art de bâtir par le ciment armé» ${ }^{14}$ garantit la solution de la «respiration à l'intérieur de l'édifice». ${ }^{145}$

En outre la conjonction du «brevet Gustave Lyon (aération ponctuelle) et du brevet Le Corbusier et Pierre Jeanneret (murs neutralisants), transforme du tout au tout les traditions du chauffage des immeubles». ${ }^{146}$

Selon Zervos telles méthodes de construction «entraînent des conséquences architecturales impératives», ${ }^{147}$ quel la réalisation de bâtiments type, par exemple 'type bureau', 'type assemblée' et «leur enveloppe est réduite à des plans lisses (doubles membranes). Ces plans sont tout de verre, tout de pierre ou mixtes, suivant la destination des locaux». ${ }^{148}$

Un couple important d'éléments est constitué par le toiture «plate avec écoulement des eaux pluviales ou de fonte des neiges à l'intérieur» ${ }^{149}$ et par le soutien des «corps de batiments qui sont sur pilotis, avec circulations diverses des voitures ou des piétons au-dessous». ${ }^{150}$ De telle manière surgit «un complexe plan architectural nouveau, entièrement inédit, imposant à l'architecture un système esthétique nouveau». ${ }^{151}$

Pour Zervos «l'architecture est faite de rapports émouvants. Ce qui permet la perception des rapports, ce sont des volumes, des surfaces, des lignes limpides et lisibles clairement. Ce qui apporte l'émoi artistique c'est la qualité des rapports que ces éléments géométriques ont entre eux». ${ }^{152}$

Zervos dans les Cahiers d'art de quelque an après, remarque que cependant le «Conseil du Bâtiment du Palais des Soviets a pris dernièrement la décision de faire exécuter le projet élaboré par des architectes russes», ${ }^{153}$ et conclut l'article en écrivant que «si nous approuvons l'organisation intérieure de ce palais, nous élevons absolument contre son expression plastique». ${ }^{154} \mathrm{Il}$ comprenait que les Soviet eussent voulu ériger en hauteur un monument pour commémorer les luttes et la victoire du prolétariat russe, mais il ne se comprennais pas comme, pour les exprimer, les architectes n'eussent pas été capables de trouver autre chose que des souvenirs archéologiques sans importance.

\footnotetext{
143 Ibidem.

${ }^{144}$ Ibidem.

${ }^{145}$ Ibidem.

${ }^{146}$ Ivi, p. 163.

${ }^{147}$ Ibidem.

${ }^{148}$ Ibidem.

${ }^{149}$ Ibidem.

${ }^{150}$ Ibidem.

${ }^{151}$ Ibidem.

152 Ibidem.

${ }^{153}$ Zervos C., Le futur palais des Soviets à Moscou, «Cahiers d'art, Revue paraissant dix fois pas an. Directeur: Christian Zervos. Peinture, Sculpture, Architecture, Art ancien, Ethnograpgie, Cinéma», n¹-4, Paris, 1934, p.114.

${ }^{154}$ Ibidem.
} 


\section{Bibliography/references}

Giedion, Siegfried, "Le Corbusier et l'architecture contemporaine par S.Geidion", in Cahiers d'art, Revue d'art paraissant dix fois par an- Directeur: Christian Zervos. Peinture- sculpture-Architecture-Art ancienethnographie-cinéma, IV, Paris, 1930

Giedion, Siegfried, "Le problème du luxe dans l'architecture moderne. A propos d'une nouvelle construction a Garches de Le Corbusier et Pierre Jeanneret", in Cahiers d'art, Revue d'art paraissant dix fois par anDirecteur: Christian Zervos. Peinture- sculpture-Architecture-musique-mise en scène-disques-cinéma, V-VI, Paris, 1928

Le Corbusier, "La Salle Pleyel. Une preuve de l'évolution architecturale", Cahiers d'art, Revue d'art paraissant dix fois an-Directeur: Christian Zervos. Peinture- sculpture-Architecture-musique-mise en scène-disquescinéma, II, Paris, 1928

Le Corbusier, "Maison de l'Union des cootives de l'U.R.S.S. a Moscou par Le Corbusier et Pierre Jeanneret", Cahiers d'art, Revue d'art paraissant dix fois par an- Directeur: Christian Zervos. Peinture- sculptureArchitecture-musique-mise en scène, IV, Paris, 1929

Le Corbusier, "Projet pour la construction du palais des Soviets par Le Corbusier et Pierre Jeanneret", in Cahiers d'art, Revue paraissant dix fois pas an - Directeur: Christian Zervos - Peinture, Sculpture, Architecture, Art ancien, Ethnograpgie, Cinéma, Editions «Cahiers d'art», 14, rue du Dragon, Paris (IV arrondissement), I-II, Paris, 1932

Le Corbusier, Notes à la suite, «Cahiers d'art, Bulletin mensuel d'actualité artistique publié sous la direction de Christian Zervos», III, Paris, 1926

Zervos, Christian, "Immeuble «Clarté» a Genève par Le Corbusier et Pierre Jeanneret", in Cahiers d'art, Revue paraissant dix fois pas an - Directeur: Christian Zervos - Peinture, Sculpture, Architecture, Art ancien, Ethnograpgie, Cinéma, Editions «Cahiers d'art», 14, rue du Dragon, Paris (IV arrondissement), VI-VII, Paris, 1932

Zervos, Christian, "Jeunes architectes. A propos de leur ex position à la Galerie des "Cahiers d'art" Février-Mars 1935", in Cahiers d'art, Revue paraissant dix fois pas an-Directeur: Christian Zervos - Peinture, Sculpture, Architecture, Art ancien, Ethnograpgie, Cinéma, I-IV, Paris, 1935

Zervos, Christian, "Le futur palais des Soviets à Moscou", in Cahiers d'art, Revue paraissant dix fois pas an Directeur: Christian Zervos- Peinture, Sculpture, Architecture, Art ancien, Ethnograpgie, Cinéma, I-IV, Paris, 1934

Zervos, Christian, "Pour la création a Paris d'un Musée des artistes vivants", Cahiers d'art, Revue d'art paraissant dix fois par an- Directeur: Christian Zervos. Peinture- sculpture-Architecture-Art ancienethnographie-cinéma, I, Paris, 1930

Zervos, Christian, "Pour la création a paris d'un musée des artistes vivants(II). Réponse et projet d'aménagement et d'organisation, par Le Corbusier et Pierre Leanneret", Cahiers d'art, Revue d'art paraissant dix fois par anDirecteur: Christian Zervos. Peinture- sculpture-Architecture-Art ancien-ethnographie-cinéma, ${ }^{\circ} 1$, Paris, 1931

Zervos, Christian, "Qui batira le palais des nations a Genève? Étude-manifeste de «Cahiers d'art» en faveur d'un projet moderne", Cahiers d'art, Peinture, sculpture, Architecture, musique. Directeur: Christian Zervos, IX, Paris, 1927

Zervos, Christian, "Qui batira le Palais des Nations? II. La situation actuelle", Cahiers d'art, Peinture, sculpture, Architecture, musique. Directeur: Christian Zervos, IX, Paris, 1927

Zervos, Christian, "Qui batira le Palais des Nations? III. Decisione del jury", Cahiers d'art, Peinture, sculpture, Architecture, musique. Directeur: Christian Zervos, n 9, Paris, 1927

Zervos, Christian, "Qui batira le Palais des Nations? IV. Le Conseil des Nations va statuer", Cahiers d'art, Revue d'art paraissant dix fois an-Directeur: Christian Zervos. Peinture-sculpture-Architecture-musique-mise en scène-disques-cinéma, II, Paris, 1928 
Zervos, Christian, "Réalisations et projets. Le Corbusier et Pierre Jeanneret. Intérieurs", Les arts de la maison, Printemps \& été, Éditions Albert Morancé, Paris, 1926

Zervos, Christian, "Un projet de centre mondial a Genéve", Cahiers d'art, Revue d'art paraissant dix fois par an-Directeur: Christian Zervos. Peinture-sculpture-Architecture-musique-mise en scène-disques-cinéma, VII, Paris, 1928

Zervos, Christian, Architecture intérieure enquetes, «Cahiers d'art, Bulletin mensuel d'actualité artistique publié sous la direction de Christian Zervos», $\mathrm{n}^{\circ} 1$, janvier, Paris, 1926

Zervos, Christian, Projet pour le palais de la S.D.N. à Genève, par Le Corbusier et Pierre Jeanneret, «Cahiers d'art, Peinture, sculpture, Architecture, musique. Directeur: Christian Zervos», IV-V, Paris, 1927

Zervos, Christian, Réalisations et projets. Le Pavillon de l'Esprit Nouveau, «Les arts de la maison», Automne \& hiver, Éditions Albert Morancé, Paris, 1925, pp. 15-18; ZERVos C., "La leçon de l'Exposition des arts décoratifs et industriels de 1925”, Les arts de la maison, Automne \& hiver, Éditions Albert Morancé, Paris, 1925 